\title{
An MRD-CI study of low-lying electronic states in $\mathrm{CaF}$
}

\author{
Peter Bündgen ', Bernd Engels and Sigrid D. Peyerimhoff \\ Institut für Physikalische und Theoretische Chemie, Universität Bonn, Wegelerstrasse 12, W-5300 Bonn 1, Germany
}

Received 22 October 1990

\begin{abstract}
Dipole moments and various spectroscopic constants of some low-lying electronic states of the $\mathrm{CaF}$ molecule have been calculated using the multireference single- and double-excitation configuration-interaction (MRD-CI) method. The electronic structure of the highly ionic molecule in various excited states can be explained in terms of different polarisations of the mainly $\mathrm{Ca}$ centered valence electron in the field of the $\mathrm{F}^{-}$anion. Plots of natural orbitals occupied by the valence electron in the different states give a qualitative picture of the charge distribution and provide a visualisation of the different polarisations of the valence electron in the various states. Comparisons with the electrostatic polarisation model of Törring, Ernst and Kändler (TEK model) are made. The unknown $\mathrm{A}^{\prime 2} \Delta$ state is predicted to lie about $21200 \mathrm{~cm}^{-1}$ above the ground state.
\end{abstract}

\section{Introduction}

In recent years, the application of high-resolution laser spectroscopy and molecular-beam techniques in the investigation of alkaline earth monohalides has provided a large amount of precise and detailed spectroscopic information [1]. The experimental data now available stimulated not only the application of various quantum-chemical methods in this field of research but also provided the basis for the development of simple semiclassical ionic-bond models which have been used successfully to describe and to predict many of the special features of group Ila monohalides. In a semiclassical approach, the electronic structure of the highly ionic alkaline earth monohalides is usually seen as a result of the mutual electrostatic interaction of a closed shell $\mathrm{X}^{-}$ halogenide anion, a closed shell $\mathrm{Me}^{2+}$ alkaline earth metal cation and an unpaired valence electron which is assumed to be mainly metal centered but strongly polarised in the field of the halogenide anion.

One of the ionic-bond models using this approach is the electrostatic polarisation model of Törring, Ernst and coworkers [2] (TK model). This model

\footnotetext{
1 Present address: University of New Brunswick, Department of Chemistry, Bag Service Number 45222, Fredericton, New Brunswick, Canada E3B 6E2.
}

has recently been extended and has been used even for treating low-lying excited states of the alkaline earth monohalides [1]. The model predicts energies and electric dipole moments for all $\mathrm{Ca}, \mathrm{Sr}$, and $\mathrm{Ba}$ monohalides in good agreement with experimental data. It is the goal of this Letter to give a comparison of results and their explanations provided by the TEK model and by large scale ab initio calculations using the MRD-CI package [3], while focusing on the charge distribution and the electronic dipole moments of some low-lying electronic states of the $\mathrm{CaF}$ molecule.

The $\mathrm{CaF}$ molecule was chosen as a test system since, for this molecule, precise experimental data are available even for the excited states. Dipole moments of the $X^{2} \Sigma^{+}(3.07 D)$ [4], $A^{2} \Pi(2.45 D)$ [5], $C^{2} \Pi(9.24 D)$ [5] and recently of the $B^{2} \Sigma^{+}$ (2.07 D) [6] electronic states have been experimentally observed by Stark spectroscopy. The electrostatic polarisation model and a more sophisticated ligand-field approach [7] have been used 10 give predictions of dipole moments and other properties of various low-lying electronic states of the $\mathrm{CaF}$ system. $\mathrm{Ab}$ initio calculations [8,9] for the $\mathrm{X}^{2} \Sigma^{+}$ ground state have been published but, to the best of our knowledge, no ab initio studies involving excited states have been reported so far.

The present study presents ab initio calculations 
for the $X^{2} \Sigma^{+}, A^{2} \Pi, B^{2} \Sigma^{+}, A^{\prime 2} \Delta, C^{2} \Pi$ and $D^{2} \Sigma^{+}$ electronic states. For the $A^{\prime}{ }^{2} \Delta$, which has not been observed experimentally (to date), predictions for the excitation energy and the dipole moment are made.

\section{Technical details}

The AO basis set employed in the present calculations consists of 80 contracted Cartesian Gaussian orbitals. A (14s, 9p) Ca basis given by Wachters [10] contracted to $[8 s, 5 p]$ was augmented with two additional $p(0.059,0.01)$ and three contracted $(5 d) /$ [3d] d functions [11] [(4.4180, 1.3088, 0.3877), $0.1148,0.0340]$ as well as with one $f(0.2)$ function (exponents are given in parentheses). For fluorine, the Huzinaga [12] $(10 s, 6 p) /[5 s, 3 p]$ contracted basis set [13] was enlarged by one additional $p$ $(0.0749)$ negative-ion function and one contracted (2d)/[1d] d function $(3.559,0.682)$. Hence, the calcium atom is represented in a $[8 \mathrm{~s}, 7 \mathrm{p}, 3 \mathrm{~d}, 1 \mathrm{f}] \mathrm{ba}-$ sis set, while a [ $5 s, 4 p, 1 d]$ basis is used for the description of fluorine. To improve the convergence of Cl wavefunctions, each state was calculated within a one-particle basis which was optimized for the given state. Canonical SCF orbitals from an SCF calculation of the given state were used for the $\mathrm{X}^{2} \Sigma^{+}, \mathrm{A}^{2} \Pi$ and $A^{\prime}{ }^{2} \Delta$ while higher electronic states were calculated within a natural orbital (NO) basis. The effects of the various treatments are given elsewhere [14]. In order to account for correlation effects, multireference configuration-interaction wavefunc- tions were employed using the MRD-CI package [3]. In the $\mathrm{Cl}$ calculations, the seven valence electrons on fluorine and the electrons of the $M$ and $N$ shell of calcium are included in the correlation treatment. Hence, a total of 17 electrons are correlated in our calculations. For the $\mathrm{X}^{2} \Sigma^{+}$state, table 1 shows a comparison of our calculated spectroscopic parameters with those obtained in previous calculations by Langhoff, Bauschlicher, Partridge and Ahlrichs (LBPA) [9]. In their calculations, calcium is described by a large (10s8p4d2f) STO basis set. For fluorine, a (6s5p4d2f) STO basis was used. It is seen that the basis set used in the present work seems to be sufficient. Taking higher than double excitations into account seems to be more important than using a larger basis set.

\section{Results and discussion}

Table 2 compares the calculated and observed spectroscopic parameters of the lowest-lying electronic states of the $\mathrm{CaF}$ molecule including the results for $\mathrm{A}^{\prime 2} \Delta$ which has not yet been observed. All $T_{e}$ values calculated for the excited states shown in table 2 are higher than the experimental ones. The deviation from experiment is smallest for $A^{2} \Pi(0.15$ $\left.\mathrm{eV} / 1186 \mathrm{~cm}^{-1}\right)$ and highest for $C^{2} \Pi(0.37 / 2549$ $\mathrm{cm}^{-1}$ ). It increases with the amount of d orbital participation in the different states (the Mulliken population analysis of the different states can be seen in figs. I and 2). The problems in calculating excitation energies in molecules containing earth alkaline at-

Table 1

Comparison of the calculated spectroscopic parameters of the $\mathrm{X}^{2} \Sigma^{+}$ground state with values from Langhoff, Bauschlicher, Partridge and Ahlrichs (LBPA) [9].

\begin{tabular}{|c|c|c|c|c|c|c|}
\hline & $x^{2} \Sigma^{+}$ & SCF & SDCI & $\mathrm{MRD}-\mathrm{Cl}$ & CPF & Exp. \\
\hline$R_{e}(\mathrm{au})$ & $\begin{array}{l}\text { LBPA } \\
\text { this work }\end{array}$ & $\begin{array}{l}3.740 \\
3.843\end{array}$ & 3.713 & 3.733 & 3.716 & $3.689^{\mathrm{c}]}$ \\
\hline$\omega_{c}\left(\mathrm{~cm}^{-1}\right)$ & $\begin{array}{l}\text { LBPA } \\
\text { this work }\end{array}$ & $\begin{array}{l}591 \\
559\end{array}$ & 587 & 581 & 583 & $588.6^{d)}$ \\
\hline$\mu$ (D) & $\begin{array}{l}\text { LBPA } \\
\text { this work }\end{array}$ & $\begin{array}{l}2.659^{\wedge \prime} \\
2.73\end{array}$ & $2.590^{\circ)}$ & $3.01^{\text {b) }}$ & $3.060^{21}$ & $3.08^{\circ)}$ \\
\hline
\end{tabular}

") Evaluated for $R=3.717$ bohr. b) Evaluated for experimental $R_{\mathrm{e}}=3.689$, see ref. [15].

c) Ref. [15]. d) Ref. [16]. ") Ref. [17]. 
Table 2

Spectroscopic parameters of lowest-lying electronic states of $\mathrm{CaF}$ in comparison with experimental values

\begin{tabular}{|c|c|c|c|c|c|c|c|c|}
\hline \multirow[t]{2}{*}{ State } & \multicolumn{2}{|c|}{$T_{\mathrm{c}}\left(\mathrm{cm}^{-1}\right)$} & \multicolumn{2}{|c|}{$R_{\mathrm{e}}(\mathrm{au})$} & \multicolumn{2}{|c|}{$\omega_{\mathrm{s}}\left(\mathrm{cm}^{-1}\right)$} & \multicolumn{2}{|c|}{$B_{\mathrm{e}}\left(\mathrm{cm}^{-1}\right)$} \\
\hline & calc. & exp. & calc. & exp. & calc. & exp. & calc. & exp. \\
\hline$X^{2} \Sigma^{+}$ & 0 & 0 & 3.733 & $3.689^{\wedge 1}$ & 581.2 & $588.6^{\circ 1}$ & 0.335 & $0.344^{\circ)}$ \\
\hline$A^{2} \Pi$ & 17712 & $16526^{\text {b) }}$ & 3.699 & $3.689^{\circ)}$ & 579.9 & $586.8^{b /}$ & 0.342 & $0.343^{b)}$ \\
\hline$B^{2} \Sigma^{+}$ & 20069 & $\mid 8841^{\circ 1}$ & 3.736 & $3.695^{81}$ & 551.5 & $572.4^{-1}$ & $(0.335)$ & $0.343^{41}$ \\
\hline$A^{\prime}{ }^{2} \Delta$ & 24851 & - & 3.776 & - & 558.9 & - & 0.328 & - \\
\hline $\mathrm{C}^{2} \Pi$ & 32765 & $30216^{c}$ & 3.804 & $3.80^{c)}$ & 518.5 & $481.7^{b)}$ & 0.323 & $0.324^{c)}$ \\
\hline$D^{2} \Sigma^{+}$ & 32741 & $30772^{b)}$ & 3.652 & - & 595.6 & $650.7^{b)}$ & 0.350 & - \\
\hline
\end{tabular}

' Ref. [15]. 'b) Ref. [16]. " Ref. [17].
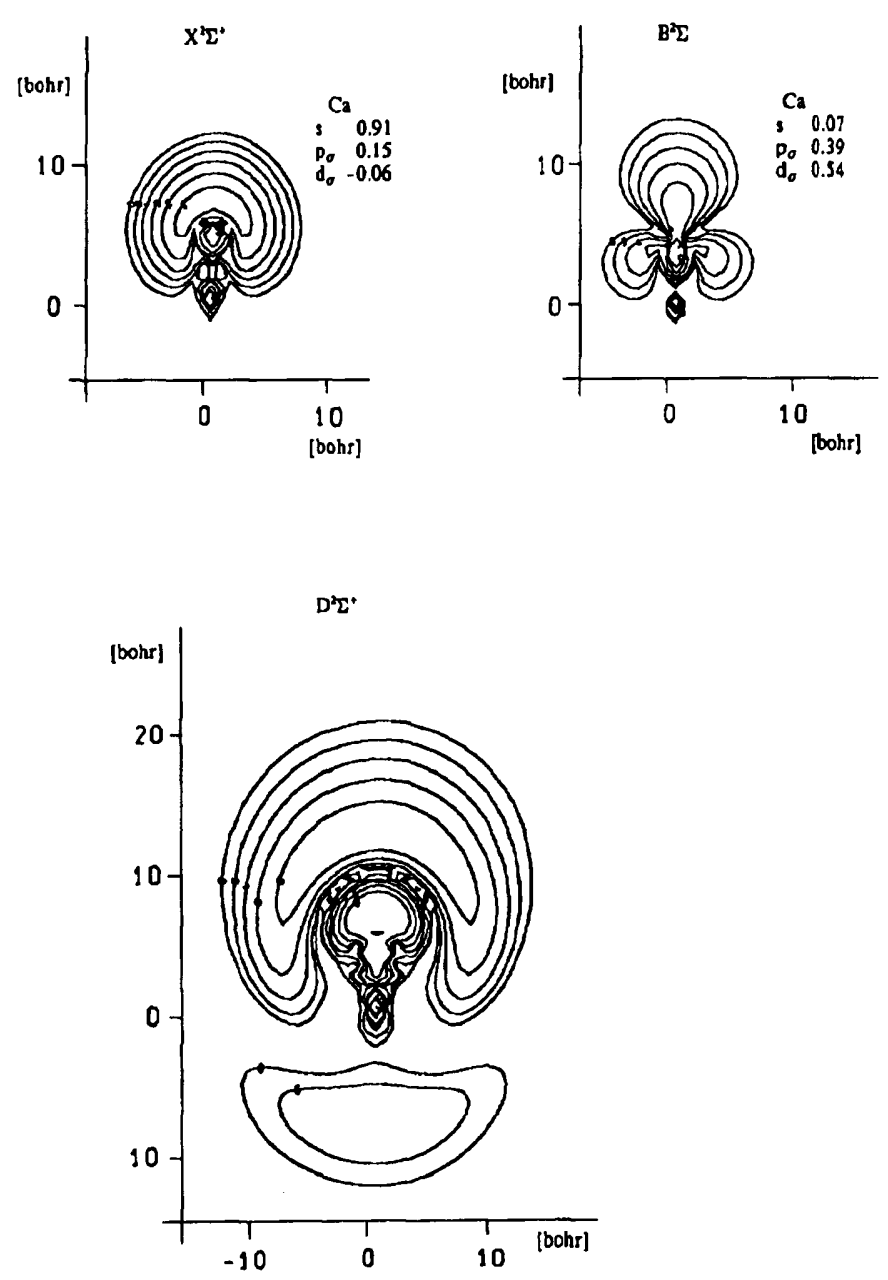

Fig. 1. Charge-density plots for the $\Sigma$ states. Shown are the singly occupied natural orbitals of each state obtained from its own CI wavefunction. The molecule is oriented with fluorine at the origin and $\mathrm{Ca}$ at an internuclear distance of +3.69 bohr. The first contour line indicates $1 / 3$ of the maximum density, consecutive lines are obtained by a factor of $1 / \sqrt{10}$. The Mulliken population analyses of $\mathrm{X}^{2} \Sigma^{+}$and $\mathrm{B}^{2} \Sigma^{+}$are given. Since the $\mathrm{D}^{2} \Sigma^{+}$possesses marked diffuse character, no population analysis was performed. 

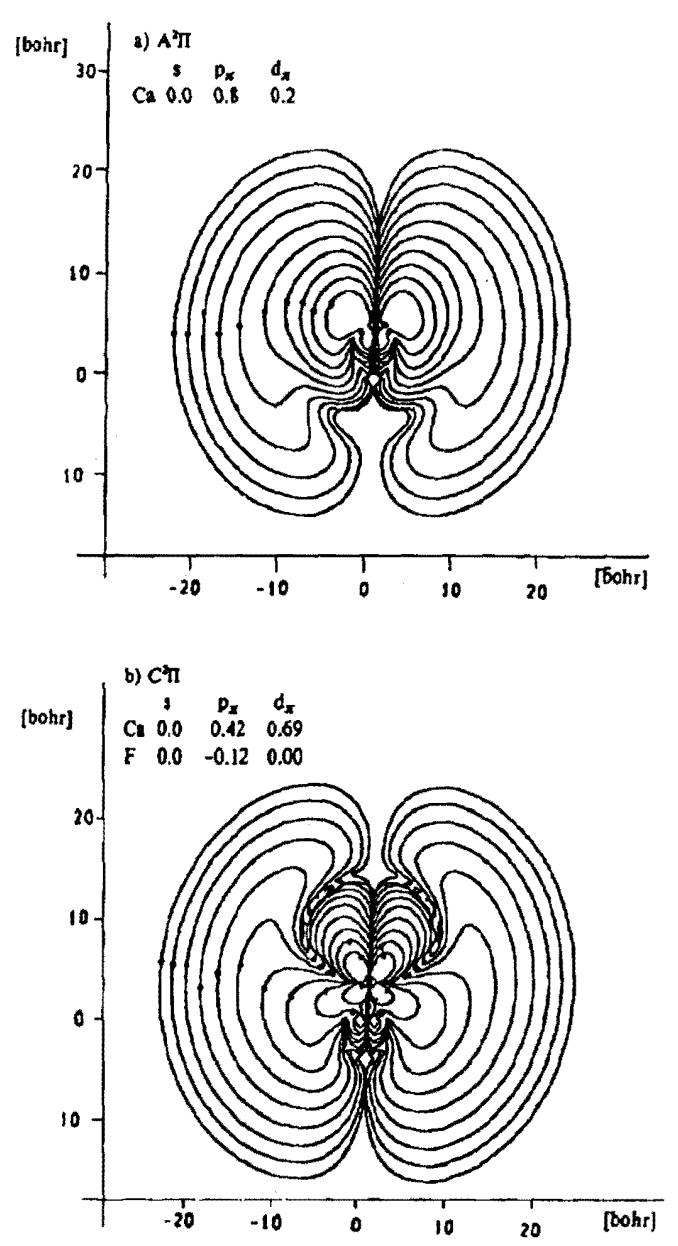

Fig. 2. Charge-density plots for the $A^{2} \Pi$ (a) and $C^{2} \Pi$ (b). For further explanation, see fig. 1 .

oms are well known $[18,19]$ and can mostly be reduced to deficiencies in the description of the earth alkaline atom. When the $\mathrm{Ca}$ basis set is applied to the $\mathrm{Ca}$ atom, the first ${ }^{3} \mathrm{D}$ atomic state is calculated to be about $0.46 \mathrm{eV} / 3710 \mathrm{~cm}^{-1}$ too high, while the first ${ }^{3} \mathrm{P}$ state was obtained within an error of only $0.07 \mathrm{eV}$. The reason for this behaviour is twofold: First, the basis set used in the present work is not flexible enough for describing the $D$ states but even a large [10s9p5d2f] STO basis set only reduces the error to about $0.1-0.2 \mathrm{eV}$ [18]. Partridge et al. [18] found that the situation improves further if the inner-shell correlation is not taken into account, but such a treatment would lead to bond distances which are much too long $[8,19]$. Since the dipole moments in this particular treatment of $\mathrm{CaF}$ strongly depend on bond distance, a study of the charge distribution would be impossible in this approach. Taking into account the error made in the excitation energies of the atomic system, we expect the position of the $\mathrm{A}^{\prime 2} \Delta$ to lie about $2000-3000 \mathrm{~cm}^{-1}$ lower than calculated. The $A^{\prime}{ }^{2} \Delta$ state is unknown but it is expected to lie higher than $19500 \mathrm{~cm}^{-1}$ [20] according to existing experimental evidence. The TEK model predicts a $T_{\mathrm{e}}$ value of about $20000 \mathrm{~cm}^{-1}$ but it is known to underestimate the excitation energies of the higher states [17]. Hence, the best estimate for the position of the ${ }^{2} \Delta$ state is around $22000 \mathrm{~cm}^{-1}$ on the basis of our present error evaluation.

For the $\mathrm{X}^{2} \Sigma^{+}$ground state, our bond length error is about 0.04 au. The calculated $R_{\mathrm{c}}$ values of the excited states are in better agreement. The calculated values for $\omega_{\varepsilon}$ and $B_{\mathrm{e}}$ also agree well (see table 2) with experimental data.

It is well known that calculated bond distances for molecular systems containing alkaline earth atoms are much too long when the metal $(n-1)$ shell is not correlated $[8,19]$ (in the $\mathrm{Cl}$ treatment). Valence electron single- and double-excitation CI (SDCI) calculations performed for the $X^{2} \Sigma^{+}$ground state of $\mathrm{CaCl}$ produced $R_{e}$ values which are 0.19 au longer than the corresponding experimental values [11]. Therefore, the $3 \mathrm{~s}$ and $3 \mathrm{p}$ electrons of calcium were included in our correlation treatment. However, similar to $\mathrm{CaCl}$ results, even the inclusion of the $(n-1)$ shell of the metal (at the SDCl level) is shown to yield bond lengths for the $\mathrm{CaF}$ molecule which are still somewhat larger than the experimental ones. For $\mathrm{CaCl}$, a bond length error of $0.049 \mathrm{au}$ was obtained at this level of theoretical treatment [11]. Similar results were also reported for $\mathrm{CaH}$ $\left(\mathrm{X}^{2} \Sigma^{+}\right)[19]$.

The bond-contraction effect, when including correlation of the $M$ shell of the calcium atom, is mainly due to double excitations where one electron is promoted from the $M$ shell of calcium and one from the outermost shell of fluorine, since the contributions of these excitations vanish in the asymptotic limit [9]. Since these double excitations (which are called pair-pair terms) account for a relatively small amount of energy, their importance may be underestimated in the presence of the large atomic cor- 
relation present in the $M$ shell of calcium which is expected to be nearly constant and not very sensitive to bond-distance changes $[9,19]$. These pair-pair terms seem to be less important in the excited states.

Table 3 shows a comparison of our theoretical dipole moments evaluated at experimental $R_{e}$ with experimental values as well as the results provided by the TEK ionic-polarisation model [1] and those calculated with a ligand-field approach [7]. The overall agreement of our calculated dipole moments is seen to be good for the $\Sigma$ state. For the $\Pi$ states, somewhat larger deviations are found (see table 3 ). In the ionic-polarisation model $[1,2]$ of TEK the dipole moments are calculated according to

$\mu=q R_{\mathrm{e}}-\left(\mu^{+}+\mu^{-}\right)$,

as a superposition of a pure ion value produced by two point charges $q$ representing the constituent building blocks $\mathrm{Ca}^{+}$and $\mathrm{F}^{-}$of the $\mathrm{CaF}$ molecule at a distance equal to the internuclear separation $R_{\mathrm{e}}$ and the mutual-induced moments $\mu^{+}$and $\mu^{-} . \mu^{+}$is the induced dipole moment at the metal produced by the action of the polarising field of the halogenide anion, $\mu^{-}$is the induced moment at fluorine produced by the field provided by the $\mathrm{Ca}^{+}$cation. Since the induced dipole moments are dependent on the ion polarisabilities and the ion polarisability of $\mathrm{Ca}^{+}$is much larger than that of $\mathrm{F}^{-}[1]$, the contribution of the induced dipole moment at the fluoride anion, $\mu^{-}$, to the overall dipole moment is small in comparison to $\mu^{+}$and will be neglected in the following discussion.

In all states except $C^{2} \Pi, \mu^{+}$accounts for a reduction of the dipole moment from its pure ionic value of $9.38 \mathrm{D}$. The unpaired electron at the metal is po- larised away from the fluorine ion. The extent of this polarisation of the metal valence electron is different from state to state. Fig. 1 shows plots of natural orbitals occupied by the valence electron in the $\mathrm{X}^{2} \Sigma^{+}$, $\mathrm{B}^{2} \Sigma^{+}$and $\mathrm{D}^{2} \Sigma^{+}$electronic states. The molecule is oriented with fluorine at the origin and $\mathrm{Ca}$ at an internuclear distance of $+3.69 \mathrm{bohr}$. The plots nicely demonstrate the predominantly metal-centered character of the valence electron as well as the large degree of polarisation which is highest in the $D^{2} \Sigma^{+}$ state. Here the large radical extent of the metal valence electron gives rise to a very large induced moment $\mu^{+}$overcoming the pure ionic moment and yielding an overall negative moment which is calculated to be $-1.85 \mathrm{D}$. Table 3 also contains the contributions $(\langle\Phi|r| \Phi\rangle)$ that the valence orbitals (see figs. 1 and 2) make to the overall dipole moment. It is interesting to compare these contributions with the values of $\mu^{+}$provided by the ionic-polarisation model [1]. It is seen that both are comparable in magnitude and show similar trends. This, and the fact that for the calculations of dipole moments $\mathrm{Cl}$ effects are very small if an appropriate one-particle basis set is used [14], explain the surprisingly good agreement of the dipole moments provided by this simple electrostatic model and experiment.

The large difference between the dipole moments of the two ${ }^{2} \Pi$ states has already been explained by assuming an opposite polarisation of the valence electron in both states [5]. Fig. 2 shows the natural orbital occupied by the valence electron in the $A^{2} \Pi$ and the $C^{2} \Pi$ state, respectively. It can be seen that in $C^{2} \Pi$, the valence electron is polarised towards the

Table 3

Comparison of the calculated dipole moments (in D) with experimental values and results provided by the ionic-polarisation model of Törring, Ernst and Kändler $[2,5,17]$ (TEK) and a ligand-field approach [7]

\begin{tabular}{|c|c|c|c|c|c|c|c|}
\hline State & Experimental & Calculated ") & $\langle\Phi|r| \Phi\rangle^{b}$ & TEK & $\mu^{+}$ & Ligand field & $\mu^{+}$ \\
\hline$X^{2} \Sigma^{+}$ & $3.07^{\mathrm{c}}$ & 3.01 & 4.41 & 3.34 & 4.68 & 3.00 & 5.03 \\
\hline$A^{2} \Pi$ & $2.45^{d)}$ & 2.83 & 4.09 & 2.57 & 5.42 & 4.09 & - \\
\hline$B^{2} \Sigma^{+}$ & $2.07^{\circ)}$ & $2.2 !$ & 4.93 & 1.59 & 6.58 & $5.7 !$ & - \\
\hline$A^{\prime 2} \Delta$ & - & 7.37 & 0.0 & 7.73 & 0.0 & 7.57 & - \\
\hline$C^{2} \pi$ & $9.24^{d)}$ & 10.15 & -2.97 & 9.21 & -0.89 & 8.23 & - \\
\hline$D^{i} \Sigma^{+}$ & - & -1.85 & 8.73 & - & - & - & - \\
\hline
\end{tabular}

"Calculated at the experimental internuclear distance. b) Contribution of the metal valence electron.

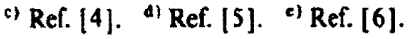


Table 4

Calculated dipole moments (in D) as a function of the internuclear distance $R$ (bohr)

\begin{tabular}{lrrrrr}
\hline & $R$ \\
\cline { 2 - 6 } & \multicolumn{1}{l}{3.5} & \multicolumn{1}{l}{3.69} & \multicolumn{1}{c}{3.8} & \multicolumn{1}{c}{4.0} & \multicolumn{1}{c}{4.2} \\
\hline $\mathrm{X}^{2} \Sigma^{+}$ & 2.35 & 2.93 & 3.26 & 3.89 & 4.51 \\
$\mathrm{~A}^{2} \Pi$ & 2.49 & 3.00 & 3.33 & 3.87 & 4.45 \\
$\mathrm{~B}^{2} \Sigma^{+}$ & 1.32 & 2.21 & 2.70 & 3.54 & 4.35 \\
$\mathrm{~A}^{\prime}{ }^{2} \Delta$ & 6.43 & 7.10 & 7.47 & 8.11 & 8.74 \\
$\mathrm{C}^{2} \Pi$ & 9.51 & 9.89 & 10.15 & 10.58 & 11.08 \\
$\mathrm{D}^{2} \Sigma^{+}$ & -2.04 & -1.81 & -1.67 & -1.37 & -1.05 \\
\hline
\end{tabular}

$F^{-}$anion. The induced moment $\mu^{+}$and $\langle\Phi|r| \Phi\rangle$ are negative and small in this state. The resulting overall dipole moment is nearly equal to the pure ionic value of $9.38 \mathrm{D}$.

The dipole moment of $A^{2} \Delta$ is calculated to be 7.37 $D$. In this state, the valence orbital has purely $d \delta$ character. The main part of the charge distribution is perpendicular to the bond axis resulting in a small induced moment $\mu^{+}$which diminishes the pure ionic value only to a small extent.

Table 4 gives our calculated dipole moments as a function of the internuclear separation $R$. Near equilibrium geometry the dipole moments of all states increase monotonically as a steep and nearly linear function of $R$. The slope of the functions is about 3 $D /$ bohr for all states and similar to results obtained for the $\mathrm{X}^{2} \Sigma^{+}$ground state in previous calculations at the SCF (3.15), SDCI (3.16) and coupled pair functional (2.91) level [9]. Vibrational averaging of the dipole moment function was not performed since it was already shown to be of minor influence $(0.1-$ 0.2 D) [9] and its effects are expected to be negligible compared to the errors resulting from the computational treatment.

\section{Summary}

In the present work, $T_{\mathrm{e}}$ values and dipole moments of the low-lying states of the $\mathrm{CaF}$ molecule have been studied. The calculated values agree well with experimental results. For the $\mathrm{A}^{\prime}{ }^{2} \Delta$ state which has not yet been observed, the $T_{\mathrm{c}}$ value is predicted to be about $22000 \mathrm{~cm}^{-1}$ and its dipole moment to be about $7.37 \mathrm{D}$. The reasons for the charge distri- bution found in the present calculations are similar to those given by the ionic-polarisation model of Törring, Ernst and coworkers [1,2]. More detailed analysis of the TEK model, including the hyperfine coupling constants of several states, will be presented elsewhere [21].

\section{Acknowledgement}

The authors wish to thank Professor T. Törring and Professor W. Ernst for numerous stimulating discussions. The services and computer time made available by the University of Bonn Computer Center are gratefully acknowledged.

\section{References}

[1]T. Törring, W.E. Ernst and J. Kändler, J. Chem. Phys. 90 (1989) 4927

[2] T. Törring, W.E. Errist and S. Kindi, J. Chem. Phys. 81 (1984) 4614.

[3] R.J. Buenker and S.D. Peyerimhoff, Theoret. Chim. Acta 35 (1974) 33; 39 (1975) 217;

R.J. Buenker, S.D. Peyerimhoff and W. Butscher, Mol. Phys. 35 (1978) 771;

R.J. Buenker and R. Phillips, J.Mol. Struct. 123 (1985) 291.

[4] W.J. Childs, L.S. Goodman, U. Nielsen and V. Pfeufer, J. Mol. Spectry. 86 ( 1981 ) 365.

[5] W.E. Ernst and J. Kändler, Phys. Rev. A 39 (1989) 1575.

[6] O. Knüppel, Diplomarbeit, FU Berlin (1989).

[7]S.F. Rice, H. Martin and R.W. Field, J. Chem. Phys. 82 (1985) 5023.

[8] S.R. Langhoff, C.W. Bauschlicher Jr. and H. Partridge, J. Chem. Phys. 84 (1986) 168?

[9] S.R. Langhoff, C.W. Bauschlicher Jr., H. Partridge and R. Ahlrichs, J. Chem. Phys. 84 (1986) 5025.

[10] A.J.H. Wachters, J. Chem. Phys. 52 (1970) 1033.

[11] N. Honjou, G.F. Adams and D.R. Yarkony, J. Chem. Phys. 79 (1983) 4376.

[12] S. Huzinaga, J. Chem. Phys. 42 (1965) 1293.

[13] T.H. Dunning, J. Chem. Phys. 55 (1971) 716.

[14] P. Bündgen, Diplomarbeit, Bonn ( 1989 ).

[15] M. Dulick, P.F. Bernath and R.W. Field, Can. J. Phys. 58 (1980) 703.

[16]K.P. Huber and G. Herzberg, Molecular spectra and molecular structure, Vol. 4. Constants of diatomic molecules (Van Nostrand Reinhold, New York, 1979).

[17] J. Kändler, Ph.D. Thesis, Berlin (1988).

[18] H. Partridge, C.W. Bauschlicher Jr., S.P. Walch and B. Liu, J. Chem. Phys. 79 (1983) 1866.

[19] L.G.M. Pettersson, P.E.M. Siegbahn and S. Ismail, Chem. Phys. 82 (1983) 355.

[20] M. Gründler, Diplomarbeit, Berlin (1990).

[21] P. Bündgen, B. Engels and S.D. Peyerimhoff, in preparation. 